Dermatologische Zeitschrift. 1907;14:I-IV

\title{
Contents, Vol. 14, 1907
}

Dermatologisehe Zeitsehrift.

Herausgegeben

Prof. Dr. O. LASSAR.

Band XIV.

Mit zahlreichen Abbildungen im Text und 7 Tafeln.

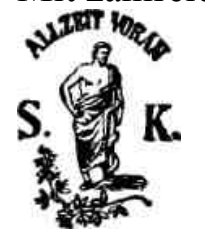

Berlin 1907.

VERLAG VON S. KARGER

KARLSTRASSE 15.

Allo Rechte vorbehalten.

Gedruckt bei Imberg \& Leîson in Berlin W.

Inhalts -Verzeichnis.

Origin al $>$ Arb $\theta$ iten.

Axmann,E., Ueber einen mittelst Uviolstrahlen behandelten

Fall von Ichthyosis diffussa 109

Becker, Ph. Ferd., Syphilitische Sehnervenentzündung in der Frühperiode 744

Blaschko, A., Was leisten die neueren Behandlungsmethoden des Lupus vulgaris, und welches sind ihre Indikationen? 1

- $\quad$ - Betrachtungen über Ichthyosis. (Hierzu Taf. VI-VII) 752

Bohaç, Karl, Beitrag zur Kenntnis des Naevus (systematisatus) ichthyosiformis 535

Burgsdorf, W·, Zur Kasuistik der idiosynkrasischen Haut-

krankheiten 675

Fellner, Otfried 0., Einige Fälle von paraurethraler

Eiterung beim Weibe 157

Fricke, H., Toxikodermie nach Röntgenbestrahlungen . . 417 Friederichs, Adolf, Drei Fälle von

Psoriasis vulgaris bei

Säuglingen 232

Grosser, Paul, Ueber Impftuberkulose

Heller, Julius, Ueber die Organisation von Heimen fur

hereditär-syphilitische Kinder497

Hesse, E, Intoxikationserscheinungen nach Anwendung $\tau$ on

Schwefelzinkpaste Ill

Kanitz, Heinrich, Ueber die interne Behandlung der 
Syphilis mit Mergal 441

Kiwull, E., Das Leprosorium bei Wenden 1896-1906 . 562 Kreibich, K., Ein Fall von

Adenocarcinoma lenticulare

capillitii 651

Lassar, 0., Der Stand der P1/8öntgentherapie 682

Lipschütz, B., Ueber die Beziehungen der Spirochaeta

pallida zum Hautpigment syphilitischer Effloreszenzen 67

- $\quad$ - Weitere Untersuchungen über Molluscum contagiosum.

(Hierzu Tafel V) 481

Mayer, Theodor, Zinkperhydrolsalben in der Dermatologie 341 Neuberger, J., Die Verhütung der

Epididymitis bei der

Behandlung der Gonorrhoe im akuten und subakuten

Stadium . . 14

v. Notthaf'ft, A., Beiträge zur Legende von der Altertums-

syphilis $\quad 603$

Photinos, G. ïh., Die Herstellung und Bedeutung der

Moulagen (farbigen Wachsabdrücke) . . . . . 131

Pink us, Felix, Zwei Fälle von Sklerödem 425

- $\quad$ - Die Histologie der deprimierten weissen Flecke bei

universellen Erythrodermien 669

- IV -

Polano,M. E., Zur Histologie cles Lichen ruber verrucosus.

(Hierzu Tafel I) 101

Polland, Rudolf, Ueber vasomotorische Reflexneurosen und

verwandte Krankheitsbilder 548

Scherber, G., Ueber Spirochaetenerkrankungen .... 88 Serra, Alberto, Untersuchungen über den

Bazillus des

Ulcus molle. (Hierzu die Tafeln III-IV) . . 281, 345

- $\quad$ - Ueber die Aetiologie des Ulcus molle 403

Solger, F. B., Zur Kenntnis des Hautfarbstoffs als Schutz-

mittel 329

- $\quad$ - Weitere Beiträge zur Bedeutung des Hautfarbstoffs

nebst Bemerkungen über Leucoderma syphiliticum . 733 Stern, Carl, Ueber Neuinfektion

Hereditärsyphilitischer

und über Reinfektion im allgemeinen 197

Stern, Carl und E. Hesse, Experimentelle und klinische

Untersuchungen über die Wirkung des ultravioletten

Lichtes (Quarzlampenlicht) : 469

Vörner, Hans, Durch Eukalyptusöl hervorgerufenes Exan-

them, das später periodisch rezidivierend wird . . 678 Werther, J.F., Dermatologische

Demonstration im Kranken-

haus Dresden-Friedrichstadt 114, 359, 500

Wolters, Max, Hydroa vacciniformis (Bazin). (Hierzu

Tafel II) 263

- $\quad-$ - Die Heilung eines Falles von primärer, aszendierender

Schleimbauttuberkulose der oberen Luftwege durch 
Jod und Q, uecksilber 556

G0sellsehättsberichte.

Bericht über die Verhandlungen des IX. Kongresses der

deutschen dermatologischen Gesellschaft

450

Berliner dermatologische Gesellschaft. Sitzung vom 11. De-

zember 1906 33

- $\quad$ - - Sitzung vom 8. Januar 1907

116

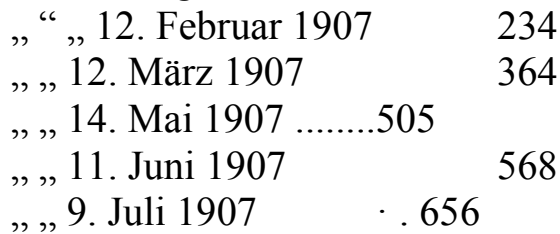

", 9 . Juli 1907

Dänische Dermatologische Gesellschaft. Sitzungen vom

Oktober 1904 bis März 1906758

Pepiodisehe Literatup . . .45, 177, 308, 376, 459, 580, 691

Buchanzeigen 64, 258, 327, 401, 529, 784

Tagresnach $\Gamma$ ichten und Notizen . . 130, 261, 402

Personalien . 534, 668

Saehregister $\quad 787$

Namenregistep $\quad 797$ 